\title{
Prediction of the Effectiveness of Spontaneous Breathing in Patients with Brain Damage of Various Etiologies
}

\author{
Yu. Yu. Kiryachkova, *, M. V. Petrova ${ }^{a}$, A. A. Loginov ${ }^{a}$, A. E. Skvortsov ${ }^{a}$, \\ K. A. Artemov ${ }^{a}$, and A. L. Parfenov ${ }^{a}$ \\ ${ }^{a}$ Federal Research and Clinical Center of Intensive Care Medicine and Rehabilitology, Moscow, Russia \\ *e-mail: kirychyu@yandex.ru
}

Received November 12, 2019; revised January 21, 2020; accepted April 19, 2020

\begin{abstract}
The aim of the study was to develop an informative method for assessing chemoreflex sensitivity and to evaluate its prognostic capacity for restoring spontaneous breathing in patients with brain damage of various etiologies. The study included 16 healthy volunteers and 38 patients on prolonged mechanical ventilation (VE) after a traumatic brain injury, anoxic brain damage, and cerebrovascular events. The external respiration variables were assessed from the initial level to the development of the first episode of desaturation with $\mathrm{spO}_{2}$ in the range of 90-80\% against the background of normobaric hypoxia as indicators reflecting the development of adaptive ventilatory response and characterizing the state of peripheral chemoreflex sensitivity (PCS). The peripheral chemoreflex sensitivity index (PCSI) was calculated using the equation: PCSI = $[R R(e): R R(i)] \times[V t(e): \operatorname{Vt}(i)] \times[V E(e): V E(i)] \times \operatorname{Vt}(e) \times \operatorname{VE}(e)$, where PCSI is the peripheral chemoreflex sensitivity index in $\mathrm{L}^{2} / \mathrm{min}$; RR(i) and $\mathrm{RR}(\mathrm{e})$; Vt(i) and $\mathrm{Vt}(\mathrm{e})$; VE(i) and VE(e) are the respiratory rate, tidal volume, minute ventilation initially (i), before a functional stress test, and during a functional test of normobaric hypoxia with $\mathrm{spO}_{2}$ in the range of $90-80 \%$ (e). With the PCSI values $\geq 15.6 \mathrm{~L}^{2} / \mathrm{min}$, successful weaning from ventilators and recovery of spontaneous breathing are predicted. The sensitivity and specificity of PCSI were 78.57 [95\% CI: 49.2-95.26] and 83.3\% [95\% CI: 62.6-95.26], respectively. The sensitivity and specificity of the traditional indicator of the success rate of weaning from ventilators and recovery of spontaneous breathing, Rapid Shallow Breathing Index (RSBI), in this cohort of patients was 69.23 [95\% CI: 38.6-90.9] and $28.0 \%$ [95\% CI: 12.03-49.3], respectively. A predictor of the patient's weaning from mechanical ventilation is the assessment of peripheral chemoreflex sensitivity, which can be measured by a simple non-invasive bedside test based on measuring the difference in external ventilation parameters before and during a functional normobaric hypoxic trial.
\end{abstract}

Keywords: peripheral chemoreflex sensitivity, predictor discontinuation of mechanical ventilation, functional stress test, recovery of spontaneous breathing

DOI: $10.1134 / \mathrm{S} 0362119720050060$

One of the most important criteria for the rehabilitation of patients after traumatic brain injury (TBI), damage to the brain of vascular origin, and perioperative neurosurgical complications is the possibility of spontaneous breathing, without the use of mechanical ventilators. According to the epidemiological data of the American Association of Surgery and Trauma, 800 thousand artificial lung ventilations (ALV) are performed in the United States per year, with an average of 2.7 episodes per 1000 inhabitants, while $20 \%$ have difficulty being disconnected from the respiratory support. Economic costs range from 600 to 1500 US dollars per day per patient or a total of 27 billion US dollars (12\% of the cost of treating a patient) [1]. The effectiveness of external and internal ventilation of a person, the possibility of spontaneous breathing, dominantly depends on the state of peripheral and central chemoreflex. The peripheral chemoreflex sensitivity is the most important homeostatic indicator of the adaptive reserves of the body, which determines the possibility or impossibility of success of taking patients off the ventilators.

The aim of the study was to develop an informative method for assessing chemoreflex sensitivity and to evaluate its prognostic capabilities for recovery of spontaneous breathing in patients with brain damage of various etiologies.

\section{METHODS}

The control group included 16 healthy volunteers (without somatic pathology) aged 22-55 years ( 8 men and 8 women; average age (mean \pm SEM), $32.2 \pm$ 1.76). The main group included 38 patients (18 men and 20 women; average age, $53.5 \pm 2.58$ years) who were in the Federal Research and Clinical Center of Reanimatology and Rehabilitation (Moscow) in 
2017-2018. The patients were studied in a period of more than 20 days for the consequences of a head injury $(n=14 ; 36.8 \%)$; the consequences of acute cerebrovascular accident (stroke) $(n=12 ; 31.6 \%)$; the effects of anoxic brain damage $(n=2 ; 5.3 \%)$; and the consequences of subarachnoid hemorrhage $(n=10$; $26.3 \%$ ). All patients were on mechanical ventilation for 21 days or longer. The criteria for the evaluation of peripheral chemoreflex sensitivity (PCS) trial were the age over 18 years and the absence of inotropes or sedatives. The exclusion criteria were closed chest injury, central motoneuron disease, massive bleeding, acute renal and hepatic failure, body temperature $>38^{\circ} \mathrm{C}$, heart rate $>140 \mathrm{bpm}$, high patient dependence on respirator $\left(\mathrm{FIO}_{2}>50 \%\right.$, PEEP $>8 \mathrm{~cm} \mathrm{H} \mathrm{H}_{2} \mathrm{O}$, lack of spontaneous inhalations). The ventilation mode in all patients is CPAP with standard respirator settings: airway pressure (PEEP), 5-8 $\mathrm{cmH}_{2} \mathrm{O}$.

The success of weaning from mechanical ventilation was defined as the lack of mechanical ventilation within 30 days after the cessation of respiratory support. Patients were examined for 60 days of intensive care and rehabilitation at the Federal Research and Clinical Center of Intensive Care Medicine and Rehabilitology, Moscow, Russia. A functional stress test was performed for healthy volunteers and patients with mechanical ventilation support using a gas hypoxic mixture for 1-3 min (ReOxy 60-2001, Bitmos, Germany). The main block apparatus produces a hypoxic gas mixture with a $10 \%$ oxygen concentration by separating atmospheric air into oxygen and nitrogen. The apparatus provides a one-way supply of the gas mixture in the direction from the installation to the patient, preventing the reverse flow of the gas mixture. An individual breathing circuit contains a face mask with an exhalation valve, a bacterial filter, a connector, and a breathing tube. In patients with a tracheostomy or endotracheal tube, a breathing circuit with an exhalation valve used in a Carina apparatus (Dräger, Germany) was employed. The principle of hypoxia under normal atmospheric pressure eliminates the possibility of decompression disorders. Based on the difference between the initial and external respiration indices during the functional test, the peripheral chemoreflex sensitivity is quantitatively determined by calculating the index of peripheral chemoreflex sensitivity (PCSI) according to the formula developed at the Federal Center for Scientific Research of the Russian Federation (patent no. 2693442 C1 of July 2, 2019 (RU)). The PCS index was calculated using the formula: PCSI $=[R R(e): R R(i)] \times[V t(e): \operatorname{Vt}(i)] \times$ $[\operatorname{VE}(\mathrm{e}): \mathrm{VE}(\mathrm{i})] \times \mathrm{Vt}(\mathrm{e}) \times \mathrm{VE}(\mathrm{e})$, where PCSI is the peripheral chemoreflex sensitivity index in $\mathrm{L}^{2} / \mathrm{min}$; $\mathrm{RR}(\mathrm{i})$ and $\mathrm{RR}(\mathrm{e})$; Vt(i) and $\mathrm{Vt}(\mathrm{e})$; VE(i) and VE(e) are respiratory rate, tidal volume, the respiratory minute volume initially (i), before the functional stress test, and during the functional test of normobaric hypoxia with $\mathrm{spO}_{2}$ in a range of $90-80 \%$ (e). Pulse oximetry
$\left(\mathrm{spO}_{2}\right)$ before and during breathing a normobaric hypoxic mixture was performed using a Dräger apparatus (Germany). To compare the success of spontaneous recovery of breathing, the traditional criterion for the elimination of respiratory failure was recorded: the Rapid Shallow Breathing Index (RSBI): $f / V t$, the respiratory rate to tidal volume ratio. All patients were assessed for the effects of neurological deficit and somatic pathology according to the standard level of consciousness scales (Glasgow Coma Scale (GCG); Four Scale; and Coma Recovery Scale, Revised (CRS-R)) and the Sequential Organ Failure Assessment (SOFA).

Statistical data processing was carried out using the MedCalc software, version 18.10.2. The differences were recognized as significant at $p \leq 0.05$. The null hypothesis was evaluated using the Pearson tests $\left(\chi^{2}\right.$, chi-square), and analysis of variance (ANOVA) of samples. To evaluate the effectiveness of the proposed method, we used the Receiver Operating Characteristic (ROC) analysis (ROC curve) with the calculation of sensitivity (Se) and specificity $(\mathrm{Sp})$ and the area under the curve (AUC).

\section{RESULTS}

The increase in external ventilation parameters in healthy individuals and patients undergoing various ventilation modes in response to the normobaric hypoxic stimulus is shown in Table 1. A significant increase in all recorded parameters is evident as the adaptive ventilatory response of the body to the $\mathrm{spO}_{2}$ decrease (Table 1). In healthy individuals, the maximum and minimum PCSI values were 870.1 and $15.6 \mathrm{~L}^{2} / \mathrm{min}$, respectively; the average value was $203.28 \pm 50.4 \mathrm{~L}^{2} / \mathrm{min}$. In patients on various mechanical ventilation modes, the maximum and minimum PCSI values were 158.19 and $1.36 \mathrm{~L}^{2} / \mathrm{min}$, respectively; the average value was $22.75 \pm 5.5 \mathrm{~L}^{2} / \mathrm{min}$ (Fig. 1). A minimal PCSI of $15.6 \mathrm{~L} / \mathrm{min}$ in healthy individuals served as the minimum value of the normal function of peripheral chemoreflex with the preservation of adaptive ventilatory response to the normobaric hypoxic stimulus. All the patients were divided into two groups according to the minimal PCSI level characteristic of healthy individuals.

Group $1(n=15)$ consisted of patients on mechanical ventilation, with $\mathrm{PCSI} \geq 15.6 \mathrm{~L}^{2} / \mathrm{min}$, similar to healthy individuals (the presence of a ventilatory response to the hypoxic test and a positive prognosis for the recovery of spontaneous breathing). The average PCSI values were $48.77 \pm 10.07 \mathrm{~L}^{2} / \mathrm{min}$ (Fig. 2). In this group of patients, successful disconnection from the ventilator and recovery of spontaneous breathing occurred in 11 patients (a truly positive result); there was no recovery of spontaneous breathing in four patients (false positive result). 
Table 1. Dynamics of external respiration during the functional 5-7-min hypoxic exercise test in healthy individuals and patients on artificial lung ventilation (ALV), $M \pm m$

\begin{tabular}{|c|c|c|c|c|c|}
\hline \multirow{2}{*}{ Study group } & \multirow{2}{*}{ Study period } & \multicolumn{4}{|c|}{ Parameter } \\
\hline & & $\mathrm{RR}, \min ^{-1}$ & $\mathrm{Vt}, \mathrm{L} / \mathrm{min}^{-1}$ & $\mathrm{VE}, \mathrm{L} / \mathrm{min}^{-1}$ & $\mathrm{spO}_{2}, \%$ \\
\hline \multirow[b]{2}{*}{$\begin{array}{l}\text { Healthy individuals, } \\
n=16\end{array}$} & Initially & $13.35 \pm 0.67$ & $0.67 \pm 0.05$ & $9.06 \pm 0.78$ & $97.5 \pm 0.3$ \\
\hline & $\begin{array}{l}\text { Hypoxic test } \\
\left(\mathrm{spO}_{2} \text { in the } 80-90 \% \text { range }\right)\end{array}$ & $15.38 \pm 0.89$ & $1.3 \pm 0.08^{* * *}$ & $19.6 \pm 1.25^{* * *}$ & $82.3 \pm 0.82^{* * *}$ \\
\hline \multirow{2}{*}{$\begin{array}{l}\text { Patients on mechani- } \\
\text { cal ventilation }\end{array}$} & Initially & $26.9 \pm 1.3$ & $0.36 \pm 0.02$ & $9.04 \pm 0.54$ & $97.2 \pm 0.41$ \\
\hline & \begin{tabular}{|l|} 
Hypoxic test \\
$\left(\mathrm{spO}_{2}\right.$ in the $80-90 \%$ range $)$
\end{tabular} & $28.4 \pm 1.4$ & $0.48 \pm 0.02^{* *}$ & $13.32 \pm 0.77^{* * *}$ & $86.1 \pm 0.74^{* * *}$ \\
\hline
\end{tabular}

${ }^{*} p<0.05 ; * *<0.01 ;{ }^{* * *} p<0.001$ with respect to the initial level (VE, L/min ${ }^{-1}$, minute ventilation; Vt, L/min, tidal volume, RR, $\mathrm{min}^{-1}$, respiratory rate) recorded initially and during breathing a hypoxic mixture for $5-7 \mathrm{~min}$.

Group $2(n=23)$ included patients on mechanical ventilation, whose PCSI during the functional stress test was less than $15.6 \mathrm{~L}^{2} / \mathrm{min}$, which was not observed in healthy volunteers (lack of a ventilatory response to the hypoxic test and a negative prognosis for the recovery of spontaneous breathing). The average PCSI values were $6.68 \pm 0.66 \mathrm{~L}^{2} / \mathrm{min}$. In this group of patients, successful disconnection from the ventilator and recovery of spontaneous breathing occurred in only three patients (false negative result). Twenty patients were not weaned from mechanical ventilation for the entire observation period (true negative result). The sensitivity of the PCSI was $78.57 \%$, indicating the prognosis of a positive result of the recovery of spontaneous breathing (95\% confidence interval (CI): 49.2-95.26), and the specificity, i.e. the prediction of a negative result of the recovery of spontaneous breathing, was $83.3 \%$ (95\% CI: 62.6-95.26). Changes in the external ventilation parameters in patients with PCSI $\geq 15.6 \mathrm{~L}^{2} / \mathrm{min}$ (positive prognosis for the recovery of spontaneous breathing) and $<15.6 \mathrm{~L}^{2} / \mathrm{min}$ (negative prognosis for the recovery spontaneous breath-

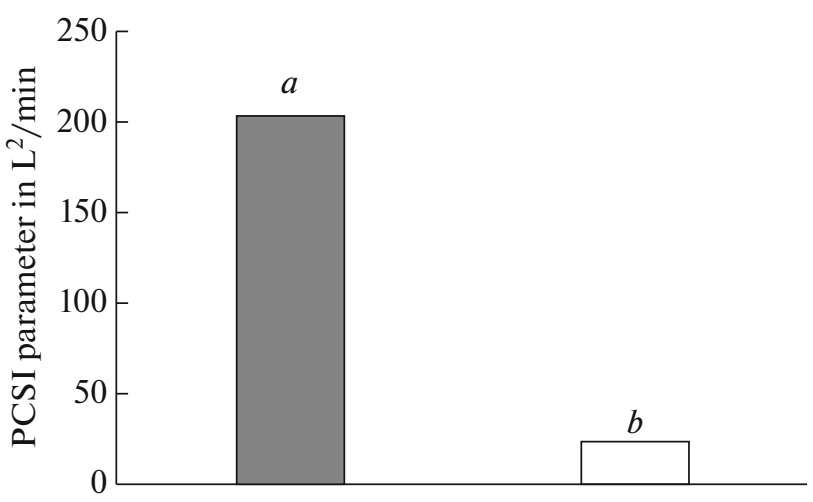

Fig. 1. Peripheral chemoreflex sensitivity index (PCSI) of healthy individuals and patients on mechanical ventilation. $a$, healthy individuals; $b$, patients on mechanical ventilation ( $p<0.001$ between $a$ and $b$ ). ing) in response to the normobaric hypoxic stimulus are given in Table 2 .

According to the RSBI as a traditional prognostic parameter of successful or unsuccessful recovery of spontaneous breathing, the following indicators were obtained in healthy individuals and patients on mechanical ventilation. The RSBI of healthy individuals varied in the $7.27-38.23 \mathrm{~min}^{-1} / \mathrm{L}$ range; the average value was $22.1 \pm 2.03 \mathrm{~min}^{-1} / \mathrm{L}$. In patients on mechanical ventilation, the range of RSBI changes was 23.1-171.27; the average value was $84.56 \pm$ $6.88 \mathrm{~min}^{-1} / \mathrm{L}$. In patients with $\mathrm{RSBI} \geq 105 \mathrm{~min}^{-1} / \mathrm{L}$ (negative prognosis for the recovery of spontaneous breathing), the average values were $139.17 \pm 6.88 \mathrm{~min}^{-1} / \mathrm{L}$. Unsuccessful recovery of spontaneous breathing was observed in seven of these patients (a truly negative result); successful recovery of spontaneous breathing was noted in four patients (false negative result). In patients whose RSBI values were $<105 \mathrm{~min}^{-1} / \mathrm{L}$ (pos-

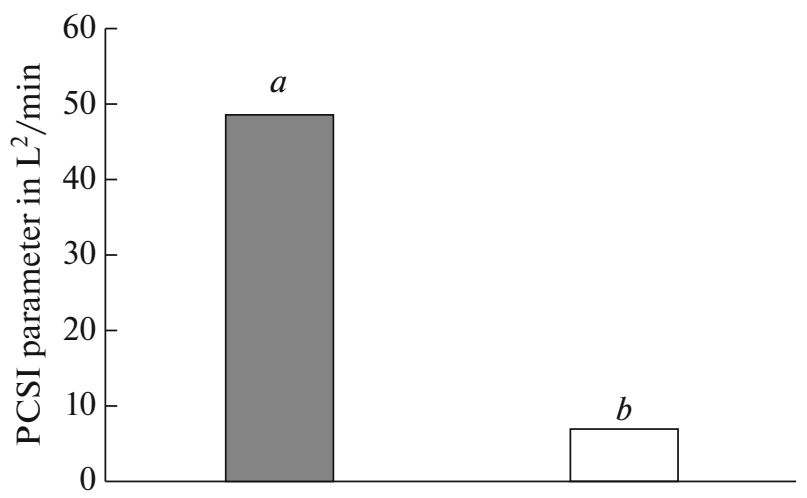

Fig. 2. Peripheral chemoreflex sensitivity index (PCSI) in patients with a positive and negative prognosis of recovery of spontaneous breathing. $a$, patients with PCSI $\geq$ $15.6 \mathrm{~L}^{2} / \mathrm{min}$ (positive prognosis for the recovery of spontaneous breathing); $b$, patients with PCSI $<15.6 \mathrm{~L}^{2} / \mathrm{min}$ (negative prognosis for the recovery of spontaneous breathing), $(p<0.001$ between $a$ and $b)$. 
Table 2. Dynamics of external respiration parameters during the functional 5-7-min hypoxic exercise test in patients with a different peripheral chemoreflex sensitivity index (PCSI), $M \pm m$

\begin{tabular}{|c|c|c|c|c|c|}
\hline \multirow{2}{*}{ Study group } & \multirow{2}{*}{ Study period } & \multicolumn{4}{|c|}{ Parameter } \\
\hline & & $\mathrm{RR}, \min ^{-1}$ & $\mathrm{Vt}, \mathrm{L} / \mathrm{min}^{-1}$ & $\mathrm{VE}, \mathrm{L} / \mathrm{min}^{-1}$ & $\mathrm{spO}_{2}, \%$ \\
\hline \multirow{2}{*}{$\begin{array}{l}\text { Group } 1 \text { with PCSI } \\
\geq 15.6 \mathrm{~L}^{2} / \mathrm{min}-\text { positive prog- } \\
\text { nosis for recovery of sponta- } \\
\text { neous breathing, } n=15\end{array}$} & Initially & $24.53 \pm 2.11$ & $0.40 \pm 0.04$ & $9.35 \pm 1.14$ & $97.5 \pm 0.3$ \\
\hline & $\begin{array}{l}\text { Hypoxic test }\left(\mathrm{spO}_{2} \text { in }\right. \\
\text { the range of } 80-90 \%)\end{array}$ & $27.79 \pm 2.3$ & $0.63 \pm 0.04^{* * *}$ & $17.1 \pm 1.29^{* * *}$ & $87.2 \pm 0.82^{* * *}$ \\
\hline \multirow{2}{*}{$\begin{array}{l}\text { Group } 2 \text { with PCSI } \\
<15.6 \mathrm{~L}^{2} / \text { min-negative prog- } \\
\text { nosis for recovery of sponta- } \\
\text { neous breathing, } n=23\end{array}$} & Initially & $28.4 \pm 1.36$ & $0.34 \pm 0.02$ & $8.96 \pm 0.59$ & $97.2 \pm 3.3$ \\
\hline & $\begin{array}{l}\text { Hypoxic test }\left(\mathrm{spO}_{2} \text { in }\right. \\
\text { the range of } 80-90 \%)\end{array}$ & $28.4 \pm 1.4$ & $0.39 \pm 0.02$ & $10.9 \pm 0.69$ & $85.6 \pm 3.1^{* * *}$ \\
\hline
\end{tabular}

*** $p<0.001$ with respect to the initial level (VE, $\mathrm{L} / \mathrm{min}^{-1}$, minute ventilation; $\mathrm{Vt}, \mathrm{L} / \mathrm{min}$, tidal volume; $\mathrm{RR}, \mathrm{min}^{-1}$, respiratory rate) recorded initially and during breathing a hypoxic mixture for 5-7 min.

itive prognosis for the recovery of spontaneous breathing), the average values were $60.47 \pm 10.68 \mathrm{~min}^{-1} / \mathrm{L}$. A successful recovery of spontaneous breathing was observed in nine patients (a truly positive result); unsuccessful recovery of spontaneous breathing was observed in 18 patients (a false positive result). The RSBI sensitivity was $69.23 \%$ (95\% CI: 38.6-90.9), and the specificity was only $28 \%$ (95\% CI: $12.03-$ 49.3). In addition, RSBI in patients with successful recovery of spontaneous breathing was $79.18 \pm$ $11.12 \mathrm{~min}^{-1} / \mathrm{L}$; in patients with unsuccessful recovery of spontaneous respiration, $90.2 \pm 6.88 \mathrm{~min}^{-1} / \mathrm{L}$; the difference was statistically nonsignificant.

When comparing group 1 and group 2 patients with $\mathrm{PCSI} \geq 15.6 \mathrm{~L}^{2} / \mathrm{min}$ (positive prognosis for recovery of spontaneous breathing) and $<15.6 \mathrm{~L}^{2} / \mathrm{min}$ (negative prognosis for recovery of spontaneous breathing), the $\chi^{2}$ test $=14.017(p<0.001)$, which allows us to reject the null hypothesis of the absence of a relationship between chemoreflex sensitivity and the possibility of patient disconnection from ventilators. At the same time, when comparing group 1 and group 2 patients, using the traditional RSBI $\geq 105 \mathrm{~min}^{-1} / \mathrm{L}$ (negative prognosis for recovery of spontaneous breathing) and $<105 \mathrm{~min}^{-1} / \mathrm{L}$ (positive prognosis for recovery spontaneous respiration), the $\chi^{2}$ test $=0.142(p>0.05)$, which does not allow us to reject the null hypothesis of the relationship between RSBI and the possibility of patient disconnection from ventilators. When comparing the variance of parameters in the PCSIselected group 1 and group 2 patients, significant differences in the group averages were also obtained, which cannot be considered accidental $(F=5.01, p<$ $0.05)$. Finally, when the ROC analysis was used and the AUC for the ROC curve was calculated, the AUC in patients with $\mathrm{PCSI} \geq 15.6 \mathrm{~L}^{2} / \mathrm{min}$ (positive prognosis for recovery of spontaneous breathing) and $<15.6 \mathrm{~L}^{2} / \mathrm{min}$ (negative prognosis for recovery of spontaneous respiration) amounted to $0.8834 \pm 0.05$, which shows a high sensitivity of the method with a binary assessment of the prognosis of weaning patients from mechanical ventilation machines. In turn, the AUC in patients according to RSBI was $0.5038 \pm 0.09$, which is actually close to a random result and does not allow a reliable prognosis of the recovery of spontaneous breathing in ventilator-dependent patients to be obtained. In groups with different chemoreflex sensitivity (PCSI $\geq 15.6 \mathrm{~L}^{2} / \mathrm{min}$, group 1 ; PCSI < $15.6 \mathrm{~L}^{2} / \mathrm{min}$, group 2), no differences in the level of consciousness determined by the standard scales (GCS, Four scale, and CRS-R), multiple organ failure (SOFA), and age were obtained, and these factors do not significantly affect the process of recovery of spontaneous breathing (Table 3 ).

\section{DISCUSSION}

The clinical approach proposed in this study for determining the readiness of weaning patients with brain injuries from mechanical ventilation is based on the assessment of a natural, phylogenetically determined, adaptive response of the body to the effect of hypoxia (reduced oxygen content in the inhaled air), a universal stressor for the human body. In this case, evaluating the PC sensitivity to hypoxia, one can obtain parameters of readiness of the human body to breathe spontaneously during artificial ventilation. It is known that the development of compensatory hyperventilation is a normal physiological adaptation response with the appearance of various types of hypoxia. The reflex appears at the birth of an individual, persists throughout life and is mainly provided by the functioning of the chemoreceptor zone of the bifurcation of the common carotid artery. This reflex is one of the most important adaptive reactions of the body, ensuring the preservation of homeostasis during critical and extreme influences. In this regard, it is extremely important to identify groups of patients on mechanical ventilation with preserved chemoreceptor 
Table 3. Scales of the level of consciousness, organ dysfunction ( $S O F A$ ), and the age of patients in groups of patients with preserved chemoreflex sensitivity (PCSI $\geq 15.6 \mathrm{~L}^{2} / \mathrm{min}$, group 1 ) and its pathological decrease (PCSI $<15.6 \mathrm{~L}^{2} / \mathrm{min}$, group 2)

\begin{tabular}{l|c|c|c}
\hline \multirow{2}{*}{ Parameter } & \multicolumn{2}{|c|}{$M \pm m$} & $\begin{array}{c}\text { Significance } \\
\text { of differences } \\
\text { between groups } \\
1 \text { and 2, } p\end{array}$ \\
\cline { 2 - 3 } & PCSI $\geq 15.6 \mathrm{~L}^{2} / \mathrm{min}$-group 1 & PCSI $<15.6 \mathrm{~L}^{2} /$ min -group 2 & $>0.05$ \\
Glasgow Coma Scale (GCS), in points & $9.73 \pm 0.58$ & $8.7 \pm 0.78$ & $>0.05$ \\
Four Consciousness Scale, in points & $11.42 \pm 0.66$ & $9.17 \pm 1.09$ & $>0.05$ \\
Coma Recovery Scale (CRS-R), in points & $9.42 \pm 1.39$ & $7.11 \pm 1.19$ & $>0.05$ \\
SOFA, in points & $2.73 \pm 0.31$ & $3.61 \pm 0.44$ & $>0.05$ \\
Age, years & $49.68 \pm 2.57$ & $55.94 \pm 3.67$ & \\
\hline
\end{tabular}

sensitivity or its pathological decrease. To date, there is no simple non-invasive test with a quantitative assessment of PCSI, which complicates the search for target therapy and prognosis of weaning patients from mechanical ventilation. Dysfunction of chemoreceptor sensitivity is manifested in two main options: (1) activation of a chemoreflex with tachypnea, the development of shallow respiration in combination with sympathetic hyperactivity and (2) deactivation (decrease in sensitivity) of chemoreflex without the development of a proper ventilatory response to the pathological development of hypoxia and hypercapnia. The situation of a PCSI decline causes severe forms of respiratory failure and prolonged and severe dependence of patients on mechanical ventilation machines [2-5]. The determination of chemoreflex sensitivity is an urgent task of modern research. Mirizzi et al. [6] used an assessment of chemoreflex in clinical settings in patients suffering from heart failure. We studied chemoreflex sensitivity peripheral to hypoxic and central to hypercapnic stimulus in 191 patients with systolic heart failure (left ventricular ejection fraction less than 50\%). As a loading effect, the rebreathing technique with the simultaneous use of hypoxia and hypercapnia was used. Clinical and neurohumoral parameters were evaluated in all patients: ECG, cardiopulmonary exercise test, respiration parameters, and the respiratory minute volume (VE) to carbon dioxide production $\left(\mathrm{VCO}_{2}\right)$ ratio during exercise. The study of these parameters indicates the activation of the chemoreceptor reflex to hypoxia and hypercapnia in patients with left ventricular failure. The use of this method allowed us to perform mixed assessment of peripheral and central chemoreceptor sensitivities using both the hypoxic and hypercapnic stimuli. Unfortunately, the authors of the method do not give the quantitative indices of the norm and pathology of chemoreceptor sensitivity necessary to solve the question of the patient readiness for being weaned from the ventilator. Zabolotsky and Trembach [7] proposed a simple and effective method for assessing PCSI. According to different durations of induced threshold apnea, a different degree of peripheral chemoreflex sensitivity is determined. However, this method for assessing peripheral chemoreflex sensitivity is difficult to perform in patients with impaired consciousness, due to the need for voluntary breathholding. In another prospective study, the authors determined central venous hemoglobin saturation $\left(\mathrm{ScvO}_{2}\right)$ as a criterion for the possibility of weaning a patient from mechanical ventilation [8]. The study included 77 patients aged $18-86$ years. All patients were switched to spontaneous breathing and at the same time were continuously monitored for $\mathrm{ScvO}_{2}$ using the method of invasive oximetry. The dynamics of $\mathrm{ScvO}_{2}$ in the parameters before the start and end of spontaneous breathing $\left(\Delta \mathrm{ScvO}_{2}\right)<4 \%$ between the beginning and the end of the study independently predicted the successful outcome of weaning from mechanical ventilation in $63.8 \%$. The technique includes a puncture of the central vein; therefore, it is invasive. It is necessary to use expensive equipment (fiberoptics). A method for predicting discontinuation of mechanical ventilation before and after breathing a hypercapnic mixture with a $10 \% \mathrm{CO}_{2}$ content until $\mathrm{PetCO}_{2}$ reaches $70 \mathrm{mmHg}$ has been proposed. As a predictor of the success of the weaning of patients from mechanical ventilation, the ratio of changes in minute ventilation $(\triangle \mathrm{VE})$ to partial carbon dioxide tension at the end of expiration $\left(\Delta \mathrm{PetCO}_{2}\right)$, the $\Delta \mathrm{VE} / \Delta \mathrm{PetCO}_{2}$ ratio, was proposed, and at a ratio of $0.40 \pm 0.16$, a successful recovery of spontaneous breathing and weaning from the ventilator occurred, but at $0.28 \pm$ $0.12 \mathrm{~L} / \mathrm{min} / \mathrm{mmHg}$, there was no recovery of spontaneous breathing $(p=0.036)$ [9]. However, with this method, there is no comparison of the results with healthy volunteers; complex equipment is necessary (changing the design of the breathing apparatus). The use of a hypercapnic stimulus allows, according to modern concepts, the evaluation of the sensitivity of central rather than peripheral chemoreflex.

Thus, the use of the chemoreflex sensitivity estimate opens up prospects for a completely new way to eliminate respiratory failure in patients. It is extremely important to identify groups of patients on mechanical ventilation with preserved chemoreflex sensitivity or its pathological decrease. 


\section{CONCLUSIONS}

The normal and pathological values of peripheral chemoreflex sensitivity have a significant correlation with a positive and negative prognosis of the recovery of spontaneous respiration of patients and disconnection from ventilation. At the PCSI values $\geq 15.6 \mathrm{~L}^{2} / \mathrm{min}$, weaning from mechanical ventilation is possible. The sensitivity and specificity of the PCSI were 78.57 [95\% CI: 49.2-95.26] and 83.3\% [95\% CI: 62.6-95.26], respectively. The sensitivity and specificity of the traditional indicator of the success rate of weaning from mechanical ventilation equipment, Rapid Shallow Breathing Index (RSBI), were 69.23 [95\% CI: 38.6-90.9] and 28.0\% [95\% CI: $12.03-$ 49.3], respectively, in this cohort of patients, which does not allow its use for reliable prediction of successful or unsuccessful recovery of spontaneous breathing. The PCS index makes it possible to successfully predict both the recovery of adequate spontaneous breathing and the inability to carry out patient disconnection from mechanical ventilation.

\section{FUNDING}

The budget of the Federal Research and Clinical Center of Intensive Care Medicine and Rehabilitation (Moscow).

\section{COMPLIANCE WITH ETHICAL STANDARDS}

Conflict of interests. The authors declare that they have no conflict of interest.

Statement of compliance with standards of research involving humans as subjects. All procedures performed in studies involving human participants were in accordance with the ethical standards of the institutional and/or national research committee and with the 1964 Helsinki Declaration and its later amendments or comparable ethical standards. Informed consent was obtained from all individual participants involved in the study. All studies were conducted in accordance with the principles of biomedical ethics formulated in the 1964 Helsinki Declaration and its later amendments and approved by the local Bioethics Committee of the Federal Research and Clinical Center of Reanimatology and Rehabilitology on May 27, 2019, protocol no. 1 (Moscow). Each study participant submitted voluntary informed consent, signed by him after explaining to him the potential risks and benefits, as well as the nature of the forthcoming study. If the patient, for some reason, could not personally sign this document when the state of the citizen did not allow him to express his will, this document was certified by three signatures of the employees of the Federal Research and Clinical Center of the Russian Federation (Moscow).

\section{AUTHOR CONTRIBUTIONS}

Yurii Yur'evich Kiryachkov: development of the goal and research method. Application of statistical data analysis methods; Marina Vladimirovna Petrova: optimization of presentation of the material, formation of the rubric for discussion of the results; Aleksei Anatol'evich Loginov: the performance of functional stress tests in patients and healthy individuals; Artem Evgen'evich Skvortsov: spirogram recording during the functional stress test; Konstantin Aleksandrovich Artemov: work with literature sources, their translation into Russian, systematization of material; Alexandr Leonidovich Parfenov: development of an algorithm for a stress test.

\section{REFERENCES}

1. Wunsch, H., Linde-Zwirble, W.T., Angus, D.C., et al., The epidemiology of mechanical ventilation uses in the United States, Crit. Care Med., 2010, vol. 38, no. 10, p. 1947.

2. Polupan, A.A., Popugaev, K.A., Oshorov, A.V., et al., Continuous mechanical ventilation in a neurosurgical intensive care unit (one-year analysis of results), Anesteziol. Reanimatol., 2010, no. 4, p. 63.

3. Tubek, S., Niewinski, P., Reczuch, K., et al., Effects of selective carotid body stimulation with adenosine in conscious humans, J. Physiol., 2016, vol. 594, no. 21, p. 6225.

4. Miller, A.J., Sauder, C.L., Cauffman, A.E., et al., Endurance training attenuates the increase in peripheral chemoreflex sensitivity with intermittent hypoxia, $\mathrm{Am}$. J. Physiol.: Regul., Integr. Comp. Physiol., 2017, vol. 312, no. 2, p. 223.

5. Mansukhani, M.P., Wang, S., and Somers, V.K., Chemoreflex physiology and implications for sleep apnea: insights from studies in humans, Exp. Physiol., 2015, vol. 100, no. 2, p. 130.

6. Mirizzi, G., Giannoni, A., Ripoli, A., et al., Prediction of the chemoreflex gain by common clinical variables in heart failure, PLoS One, 2016, vol. 11, no. 4, p. e0153510.

7. Trembach, N. and Zabolotskikh, I., Evaluation of breath-holding test in assessment of peripheral chemoreflex sensitivity in patients with chronic heart failure, Open Respir Med. J., 2017, vol. 27, no. 11, p. 67.

8. Georgakas, I., Boutou, A.K., and Pitsiou, G., Central venous oxygen saturation as a predictor of a successful spontaneous breathing trial from mechanical ventilation: a prospective, nested case-control study, Open Respir. Med. J., 2018, vol. 26, no. 12, p. 11.

9. Lee, C.S., Chen, N.H., Chuang, L.P., et al., Hypercapnic ventilatory response in the weaning of patients with prolonged mechanical ventilation, Can. Respir. J., 2017, vol. 2017, art. ID 7381424.

Translated by E. Babchenko 